The general view held by philosophers seems to be that when temperature rises the gravitative attraction changes (if at all) by a function of temperature only. This leads to the results stated in the beginning of their letter by Dr. Lindemann and Mr. Burton. But it is commonly conceded that at present there is no trustworthy theory of gravitation, so that one seerns entitled to suggest that any increment in the force may be a function of both mass and temperature. The simplest formula, that of the weighted mean temperature, brings the facts as at present known into line. It seems that we must await further data; for, of course, a fact, however slight, may shatter a theory lying in its course,

If $\mathrm{M}, m, \mu$ are the masses of earth, kilogram, and milligram, we have the cases cited thus. When $m$ alone rises in temperature the increment of its force on $\mu$ is $\mathrm{G} m \mu \alpha \mathrm{T} / d^{2}$, and on $\mathrm{M}$ is $\mathrm{Gmm \alpha \textrm {T }} / d^{2}$. The first is sensible, the second insensible, compared with the forces when cold. Again, when $\mu$ alone rises in temperature the increment of its force on $m$ is $\mathrm{G} \mu \mu \alpha \mathrm{T} / d^{2}$, but the increment of the force of $\mathrm{M}$ (considered as an aggregate of $\mu$ particles) on $m$ is $\mathrm{G} \mu \mu \alpha \mathrm{T} / d^{2}$, multiplied by

$$
\frac{\mathrm{M}}{\mu} \cdot \frac{\mathrm{M}}{\mathrm{M}+m} \cdot \frac{\mu+m}{\mu}=\mathrm{GM} m a \mathrm{~T} / d^{2} .
$$

This is identical with the increment of force on $m$ due to $M$ (considered as a whole), and is sensible compared with the force when cold. The series forms a consistent whole.

If the increments are due to radiation and resonance (see NaTuRE for July 13 last), there is an acoustical parallel. A medium fork will set up resonance to a slight degree in a large fork, to a greater degree in a small fork, all the forks having one frequency.

I cannot understand how the idea arises that action and reaction would ever, on this theory, be unequal. Let the force of $m$ on $\mathrm{M}$, both cold, be $\mathrm{F}$. When $m$ only rises in temperature the force is $\left(\mathrm{F}+f_{1}\right)$, and when $\mathrm{M}$ only rises it is $\left(\mathrm{F}+f_{2}\right), f_{2}$ being of a higher order than $f_{1}$. But in each of the three cases the attraction is mutual and equal between $\mathrm{M}$ and $m$.

As regards Cornu's results, I stated that we could "deduce nothing" from them, so there is a mis. apprehension. I made no attempt to reconcile his results with mine.

I do not consider that the indirect results obtained from Prof. Boys's research can be laid by the side of mine for comparison. In his experiment any rise of temperature would involve both masses. Supposing the increment is due to molecular or other agitation of $m$ due to radiation in my case, in his case there would be an enormously greater agitation in $m$ due to direct rise in temperature. I see no reason why this should not be one hundred times as great as the effect due to radiation. The weighted mean formula was used to account for the effects observed when either mass is heated. The case when both masses are heated is different, and has not been at present considered, the data at hand being indirect and inconclusive.

The probable result from my experiments is $a=+(\mathrm{I} \cdot 2 \pm 0.05) \times 10^{-5}$, or, as amended recently at Newcastle, $\alpha=+(\mathrm{r} \cdot 3 \pm 0.05) \times 10^{-5}$. Thus, while individual results differ among themselves, in some cases, by as much as the whole effect, yet the collective result is not weak.

I wish to express my appreciation of the generous terms used regarding my experiments by the authors.

University College, Nottingham, December I3. NO. 2462 , VOL. 987

\section{THE USE OF METEORITIC IRON BY PRIMITIVE MAN.}

METEORITES, as they reach our planet, are of three different kinds, namely, siderites, composed chiefly of nickel-iron; aerolites, composed chiefly of stony matter; and siderolites, composed of a mixture of iron and stone. The first and third only are of interest to us in this inquiry.

Great diversity of opinion has always prevailed among archæologists concerning the source of iron used in antiquity before it was intelligently produced from the ore. On the whole these opinions are about equally divided, though perhaps the majority are inclined to the verdict that meteoritic iron was not used by primitive man, for the following reasons:-First, because nearly all iron implements of antiquity extant, at least from the Old World (including the piece found in the Great Pyramid of Cheops), are of terrestrial origin; serondly, because it was believed to be nonmalleable; thirdly, because it was said to be too scarce; and fourthly, because it was argued that small fragments could not have been detached from meteoritic masses by the means available to primitive man.

There is, to the casual observer, a great deal of truth in these four arguments, and they prove that there were several sporadic sources from which the early iron was drawn; perhaps in one locality they were chiefly terrestrial, while the use of extra-terrestrial or meteoritic iron predominated in other localities.

The fact that iron, in the earliest ages and from whatever source, was extremely. rare is beyond all doubt. One might say that it was at least as rare as, and perhaps more valuable than, gold. It might therefore be asked: If meteoric iron was so rare and valuable, why was it not more carefully preserved by the people of antiquity? For, whereas we have plenty of gold objects of ancient workmanship in our museums, those of meteoritic iron are conspicuous by their absence. To this we may safely reply that the value of the meteoritic iron lay in its actual employment in the form of tools, implements, and weapons, and to have hidden such objects in treasure stores would have nullified their value, and that is one reason at least why so few were preserved. This disposes of argument number one.

We must bear in mind also that in the New World this argument does nnt apply with the same force, as we shall see that objects of meteoritic iron are not so rare there as in the Old World. The reason for this is that, whereas iron was produced in the Old World from the ore more than three thousand years ago, it was not introduced into the New World until the discovery of that continent about four hundred years ago, and it is obvious that objects more than three thousand years old have become rarer in our day than those comparatively new ones of only four hundred years ago.

A paper on this subject was contributed by the present writer at the autumn meeting of the Iron 